\title{
ECOPEDAGOGÍA PARA PRESERVAR LAS ÁREAS PROTEGIDAS Y EL PAISAJE CULTURAL CAFETERO
}

\author{
DIANA MAGALLY CORREA VALERO ${ }^{1}$
}

Recibido el 11 de diciembre de 2012 y aprobado el 18 de junio de 2013

\section{RESUMEN}

El siguiente artículo presenta avances de investigación en torno a la teoría y los métodos de educación y derecho ambiental. A partir de un estudio de caso concreto, se analizará la posibilidad de poner en práctica los conceptos de ecopedagogía, ecociudadanía y sociedad ecológica, así como interdisciplinariedad, interculturalidad y diálogo de saberes.

Los métodos utilizados son: investigación sociojurídica ${ }^{2}$, investigación acción participativa ${ }^{3}$ y método etnográfico ${ }^{4}$. El resultado describe papel activo de los estudiantes de derecho ambiental de la Universidad de Caldas, y las comunidades indígenas y campesinas del Triángulo del Café en Colombia, en el desarrollo de dichos conceptos y métodos, durante el periodo de tiempo comprendido entre febrero de 2007 y diciembre de 2010, como un aporte al fortalecimiento de la participación ciudadana, y la defensa de los derechos de las comunidades locales.

Del artículo se concluye que, a partir de la educación y el derecho ambiental se contribuirá en la preservación de la diversidad biológica y cultural del Triángulo del Café, en especial en las áreas protegidas y los resguardos indígenas, tanto que a largo plazo se deberá crear redes y políticas públicas que fortalezcan la gobernanza ambiental en el territorio.

\section{PALABRAS CLAVE}

Educación, participación ciudadana, áreas protegidas.

\section{ECO-PEDAGOGY, A WAY TO PRESERVE PROTECTED AREAS AND THE COLOMBIAN COFFEE CULTURAL LANDSCAPE}

\begin{abstract}
This article presents progress made in research regarding environmental educational theory and teaching methods as well as environmental law. Based on a specific case study, the possibility of putting into practice the concepts of eco-pedagogy, eco-citizenship and ecological societies, as well as interdisciplinarity, interculturality and knowledge dialogues, is analyzed.
\end{abstract}

The methods used are social-legal research, participatory action 
research and the ethnographic method. The result describes the active role of the environmental law students from Universidad de Caldas, and the indigenous communities and the rural populations in the Colombian Coffee Triangle in the development of such concepts and methods during the period comprised between February 2007 and December 2010, as a contribution to the strengthening of citizen participation and the defense of the rights of local communities.

The article concludes that from education and environmental law it would be possible to contribute to the preservation of the biological and cultural diversity of the Coffee Triangle, especially in the protected areas and the indigenous reservations in such a way that at a long term, networks and public policies must have to be created to strengthen environmental governance in the territory.

\section{KEY WORDS}

Ecology, education, citizen participation, protected areas.

\section{INTRODUCCIÓN}

El consumo indiscriminado de hidrocarburos, la emisión de gases de efecto invernadero, la ocupación de grandes extensiones de tierra para uso agrícola, la tala, la quema, la caza y la pesca indiscriminada, entre otras acciones de origen antrópico (Maya, 1996, p. 14-15), han desencadenado los fenómenos conocidos como cambio climático y la pérdida de diversidad biológica.

A nivel mundial, el cambio climático se manifiesta con la pérdida de los casquetes nivales, el aumento de la desertificación, el crecimiento del nivel de agua salada y la pérdida de agua dulce, el aumento de ciclones, tormentas y lluvias, entre otros efectos. Con relación a la salud de la especie humana, el cambio climático es causante de enfermedades respiratorias, cardiovasculares y parasitarias, causadas por mosquitos y otras plagas tropicales.

Las acciones antrópicas, también inciden en la pérdida de diversidad biológica, con la consecuente extinción de especies animales y vegetales, necesarias para la pervivencia de los ciclos biológicos sobre la tierra, y la cura de enfermedades.

En Colombia, los efectos del cambio climático se manifestaron en el recrudecimiento del invierno e intensas lluvias durante los años 2010 y 2011, que afectaron el sector agropecuario, la economía y la salud de miles de campesinos y ciudadanos colombianos.

"El "Índice de riesgo climático", elaborado por una organización no gubernamental europea llamada Germanwatch, señala anualmente a los países que 
necesitan fortalecer más su defensa contra tempestades, inundaciones, sequías y olas de calor que, según los científicos especializados en el estudio del clima de las Naciones Unidas, se agravarán este siglo.

Guatemala, Colombia y Pakistán fueron los países más afectados por los diferentes fenómenos climáticos en 2010, según este informe que tiene en cuenta el costo en materia de vidas humanas y pérdidas absolutas en dólares, así como el costo relativo de acuerdo con el nivel de prosperidad del país." (El Espectador, 2011)

Si bien es cierto que Colombia ha sido y seguirá siendo uno de los principales países afectados por el cambio climático, irónicamente es uno de los que tienen mayor potencial para la lucha contra éste, debido a su posición geográfica en la zona tropical, su condición de país amazónico, la riqueza en diversidad genética, de especies y ecosistemas, entre otras.

La región del Triángulo del Café ha sido afectada por el cambio climático; y se cita la disminución de la producción de café ${ }^{5}$, y los desastres ambientales, como deslizamientos, y desbordamiento de los ríos que originan accidentes, enfermedades y muertes trágicas, etc.

Es por ello que la conservación ambiental está intrínsecamente relacionada con la salud y la preservación de la especie humana sobre la Tierra en la denominada "trama de la vida", y las acciones del hombre en pro o en contra del ambiente, lo afectan a él, a otras especies y a diversos ecosistemas.

¿Cuál es la función de la educación en este contexto? ¿Existe la posibilidad de articular los procesos de educación universitaria en la conservación ambiental en el Triángulo del Café? En caso de ser posible: ¿Cuáles serían los métodos para lograr esta articulación?

\section{MATERIALES Y MÉTODO}

Esta investigación fue realizada durante el periodo comprendido entre 2007-2010, y requirió 22 salidas de campo con los estudiantes de la materia Derecho Ambiental, de Biología, Trabajo Social y Derecho, en las áreas protegidas del Triángulo del Café.

Mediante la preparación previa de talleres de educación ambiental, los estudiantes de derecho ambiental de la Universidad de Caldas, tuvieron la oportunidad de poner en práctica el diálogo de saberes con comunidades campesinas y funcionarios de las áreas 
protegidas, en torno a los temas de diversidad biológica, y cambio climático.

Por otra parte, el Semillero de Derecho Ambiental realizó 4 talleres, y trabajo de campo, con 45 líderes de las comunidades indígenas de Riosucio, en torno a los dos temas anteriores, pero con un nuevo enfoque: la protección de los derechos colectivos de los pueblos indígenas y tribales conforme con el Convenio 169 de la OIT.

A continuación, se presenta nuestra experiencia en la creación colectiva y desarrollo de trabajo de campo, con el fin de analizar si es posible que en el desarrollo de actividades pedagógicas, también se pueda apoyar el trabajo ambiental de las instituciones estatales como Parques Nacionales, y a las comunidades que habitan los Resguardos Indígenas del Triángulo del Café, como un ejemplo de ecopedagogía, ecociudadanía, y transición a una sociedad ecológica.

\section{RESULTADOS}

\section{Paisaje natural y cultural en el Triángulo del Café}

El principio de extraterritorialidad promueve "pensar globalmente y actuar localmente", e invita a analizar el potencial ambiental y cultural de la región del Triángulo del Café, así como el papel de la educación ambiental, para sus ciudadanos.

El Triángulo del Café está conformado por los departamentos de Caldas, Quindío y Risaralda, con características comunes tales como: sustrato natural, acción humana y capacidad productiva; gracias a estas particularidades, el 26 de junio de 2011 la UNESCO declaró que el paisaje cultural cafetero hace parte del patrimonio mundial de la humanidad, de la siguiente manera:

Un paisaje cultural es una parte del territorio, resultado de la acción humana y su influencia sobre factores naturales. El paisaje es el resultado de un proceso histórico natural y cultural de relaciones de una comunidad con un medio ambiente determinado.

Según UNESCO, se entiende por paisaje cultural el resultado de las actividades humanas en un territorio concreto. Los componentes que lo identifican son:

- El sustrato natural (orografía, suelo, vegetación, agua).

- Acción humana: modificación y/o alteración de los elementos naturales y construcciones para una finalidad concreta. 
- Actividad productiva (componente funcional en relación con la economía, formas de vida, creencias, cultura).(Red Alma Máter, 2010)

En medio del paisaje montañoso del Triángulo del Café, se encuentran diferentes áreas del Sistema de Parques Nacionales Naturales $\left(\mathrm{SPNN}^{6}\right)$, como lo son: PNN Los Nevados, el PNN Tatamá, el Santuario de Flora y Fauna Otún Quimbaya, entre otras.

En la región de Riosucio (Caldas), se encuentran los resguardos indígenas de la etnia Embera, su territorio es de carácter colectivo y protegido por la Constitución Política colombiana como inalienable, imprescriptible e inembargable ${ }^{7}$.

Frente a este imponente paisaje natural y cultural, los educadores universitarios son actores privilegiados en el compromiso de la preservación de las áreas protegidas y de la cultura asociada. Se analizará en el siguiente estudio de caso, la capacidad de interacción entre la universidad pública, la sociedad civil y los entes gubernamentales para la preservación del patrimonio mundial, proyectado en las figuras jurídicas de la UNESCO, las areas protegidas, y los resguardos indígenas.

\section{Reflexiones a partir del método y el trabajo de campo}

El Semillero de Derecho y Medio Ambiente, es un grupo interdisciplinario conformado por estudiantes de las áreas académicas de Biología, Sociología y Derecho, que se capacitó durante dos años en temas como protección de derechos de los pueblos indígenas y tribales, mecanismos de participación ciudadana, acciones constitucionales para la conservación ambiental y convenio de diversidad biológica.

Las reuniones continuas de trabajo permitieron una formación integral e interdisciplinaria, con sólidas bases teóricas, cuya finalidad era realizar trabajo de campo con indígenas y campesinos; la práctica enriqueció con las continuas discusiones sobre los diferentes métodos que se utilizan en las ciencias naturales y sociales, así como frecuentes debates sobre los métodos más convenientes para analizar la relación entre objetividad y subjetividad en las investigaciones desarrolladas.

Una de tales diferencias encontradas en el equipo de trabajo conformado entre los estudiantes de ciencias experimentales y ciencias sociales, fue la manera en que desde cada una de las diversas disciplinas se debería abordar el objeto de estudio.

Los biólogos manifiestan que cuando realizan trabajo de campo, deben buscar la invisibilidad para poder compilar los datos, desde su óptica ya que es necesario que el objeto de estudio, los animales o plantas investigados en su estado natural, no perciban la presencia 
del hombre. Al analizar los resultados en las ciencias naturales, priman los datos matemáticos y los informes basados en estadísticas; en general el método utilizado es el científico, con base en datos cuantitativos.

Para los estudiantes de derecho, sociología y trabajo social, la interacción con las comunidades locales de campesinos e indígenas en "el trabajo de campo, implica una interacción directa y un 'análisis de la subjetividad y la reflexividad'" (Guber, 2001, p. 19); en cada trabajo que realizan, focalizan su atención en la presentación de las investigaciones ante los pobladores, en la escritura del texto y la entrega final de resultados, tienen en cuenta el posicionamiento frente a las comunidades y la manera en que estos analizan y ven a los jóvenes investigadores de la Universidad de Caldas. Los estudiantes de ciencias sociales utilizan en la mayoría de sus actuaciones, el método cualitativo de investigación.

Para la construcción de un equipo interdisciplinario, fue necesario el diálogo constante y paciencia para poder comprender las diferentes visiones que los miembros del equipo tenían sobre el trabajo con las comunidades indígenas y campesinas.

Como resultado de esta interacción, los estudiantes de biología "humanizaron" sus investigaciones, y desde la práctica comprendieron la importancia del conocimiento de los pobladores sobre la diversidad biológica y cultural local; los investigadores en ciencias sociales, aprendieron a trabajar con datos cualitativos, y a utilizar el método científico con eficiencia.

A partir del trabajo con las comunidades indígenas y campesinas, los estudiantes crearon métodos novedosos para la presentación de los resultados: títeres, teatro, ponencias con imágenes y preguntas constantes que promovieron el análisis de la realidad, la eficacia de la política y del derecho; actividades para promover la interlocución entre sujetos que tienen una manera distinta de comprender el mundo; con el apoyo del arte, se logró un intercambio de saberes sobre el futuro en común en el Triángulo del Café y en temas de interés general para los humanos como especie.

Como metodología empleada: modelo de escuela activa en pequeños grupos de trabajo, que permitió discutir la aplicabilidad de la ley en los conflictos ambientales. Talleres de teatro y títeres, para la representación de casos jurídicos y mecanismos de participación ciudadana para la conservación ambiental. Modalidad de foro para temas complejos, tales como tala de madera, alquiler de tierras para la siembra de bosques madereros con especies exóticas, comercialización de fauna nativa, entre otros.

En el diálogo con los líderes indígenas, se verificó la tendencia a utilizar los mecanismos de resolución de conflictos para los 
problemas internos de los Resguardos, a partir del artículo constitucional de la jurisdicción especial indígena ${ }^{8}$.

Por su parte, los indígenas de Riosucio consideraron el Derecho Ambiental como una herramienta para la resolución de conflictos externos, como por ejemplo, aquellos que pueden surgir con una Corporación Autónoma Regional, o para detener la delincuencia ambiental generada por las personas ajenas al Resguardo como en el caso de la tala de bosques nativos o el comercio de fauna silvestre.

Como grupo se concluyó que el mayor aprendizaje fue para los estudiantes de la Universidad de Caldas, tal como se transcribe a continuación en palabras de los investigadores:

Los investigadores del semillero tenemos claro que, no basta con transmitir de manera pedagógica nuestros conocimientos, también tenemos que investigar durante nuestro trabajo de campo, grabar las intervenciones, realizar entrevistas semiestructuradas y aplicar el método etnográfico, así como llevar registro fotográfico de los hechos; de tal manera que podamos realizar un análisis de datos e informes escritos, que se puedan presentar a la comunidad científica. (Correa, 2009)

\section{Construcción de redes para la conservación ambiental y cultural del Triángulo del Café}

Si bien es cierto que la experiencia en el trabajo de campo fue positiva para los miembros de las comunidades campesinas e indígenas, estudiantes y funcionarios de los Parques, también fue insuficiente; el potencial emancipador del Derecho Ambiental es amplio, y la necesidad de capacitación en torno a los temas seleccionados es superior a un grupo de trabajo.

Para pensar en torno a la conservación del Triángulo del Café, será necesaria la construcción de redes entre la sociedad civil y el Estado, porque:

[...] al seguir una lógica reflexiva que fomenta el diálogo y la innovación continuos, las redes tienen el potencial de superar los dilemas regulativos que los mercados (que siguen la lógica del intercambio) y el Estado (que siguen la lógica de la autoridad) no pueden solucionar por sí mismos. (De Sousa y Rodríguez, 2007, p. 12)

Es a través del trabajo en red que las universidades de la región podrán tener un importante papel en el desarrollo de planes curriculares y proyectos, que incluyan la conservación ambiental en 
el sentido teórico y práctico, y podrán realizar el 'futurible' de la emancipación ecológica logrando consensos con base en la información común.

En consecuencia, se propone la articulación de la Red Sociojurídica del nodo Eje Cafetero, el Sistema Regional de Áreas Protegidas (SIRAP-EC ${ }^{9}$ ) y los equipos de trabajo ambiental de los Resguardos Indígenas de Riosucio, así:

La Red Sociojurídica del nodo Eje Cafetero, se encuentra conformada por universidades públicas y privadas de Manizales, Pereira, Armenia y Cartago, y cuenta con investigadores y profesores interesados en apoyar la conservación ambiental de la Región.

De igual manera, el SIRAP-EC liderado por los PNN, las $C A R^{10}$, el Instituto von Humboldt, la WWF ${ }^{11}$, y la Red Alma Máter, entre otros, están aunando esfuerzos para proteger la biodiversidad existente en las áreas protegidas del Eje Cafetero, priorizando en temas tales como reconversión ganadera, gestión integral del riesgo, hábitat sostenible y tema forestal [Red Alma Máter (s.f.), Comité Técnico Ecorregión Eje Cafetero, Acta 1 de marzo de 2007].

Como objetivos específicos, se pretende apoyar procesos pedagógicos en Derecho Ambiental, creando propuestas curriculares conjuntas para desarrollar en las universidades y propuestas abiertas de capacitación con ONG's, instituciones estatales y comunidad en general, así como realizar los análisis jurídicos y sociojurídicos de leyes y proyectos de ley existentes, que tengan relación con la conservación ambiental del Eje Cafetero.

Debido a las especificidades frente a la biodiversidad existentes en la región del Eje Cafetero, las posibilidades de trabajo mancomunado entre las universidades y los campesinos, las comunidades indígenas y negras en torno al tema ambiental; así como la oportunidad de generar nuevos conocimientos y prácticas pedagógicas en torno al concepto y la protección ambiental a partir de las universidades y centros educativos de la región.

\section{DISCUSIÓN}

Warat creó la palabra 'futurible', para describir los sucesos del futuro; un futuro que al identificarlo y describirlo puede ser cambiado. Consecuente con esta palabra, en el texto "Por quién cantan las sirenas"12 identificó los siguientes futuribles: "Extinción de la humanidad a causa de los desastres ecológicos generados por el 'turbocapitalismo' y el consumismo, radicalización de la barbarie debido al choque entre las diferentes culturas, y la emancipación 
ecológica como una posible salida a los dos escenarios anteriores" (Warat, 2004, p. 417).

El paradigma de la emancipación ecológica, también es analizado en el discurso del ecodesarrollo propuesto por el autor mexicano Leff, quien propone: "problematizar las bases de la producción, reconocer las leyes de la naturaleza, la diversidad étnica, y los nuevos modos de producción y estilos de vida en las condiciones y potencialidades ecológicas de cada región" (Leff, 2004, p. 16-17).

La emancipación ecológica es entonces, "un pacto por el predominio de los instintos de la vida sobre los instintos de la muerte" y para hacer realidad este 'futurible' emancipador, Warat propone la ecopedagogía y la ecociudadanía, tal y como las describiremos a continuación:

\section{Ecopedagogía}

Ecopedagogía: el paradigma ambiental y el paradigma ecológico presentan vínculos estrechos con la pedagogía y la política. La transformación ecológica-ambiental es ante todo, pedagógica y política. Las personas tienen que ser educadas para que puedan producir la transformación paradigmática rumbo a la autonomía. [...] El sobresalto salvador no podrá surgir a no ser de una enorme transformación de nuestras relaciones con el hombre, como con otros seres vivos y con la naturaleza. Es necesario substituir una cultura de competencia y agresión que gobierna actualmente las relaciones mundiales por una conciencia ecológica de la solidaridad. La ecopedagogía como sobresalto salvador. Un nuevo modelo social de educación. [...] La ecopedagogía afirma que la formación de un estudiante es un trabajo social que se compromete con la creatividad. Crear para tornar creativo al otro. (Warat, 2004 p. 434-437)

En el ámbito del Derecho:

[...] la metodología jurídica está llamada a dar una contribución importante a la práctica de una aplicación de un derecho flexible, confrontado a múltiples exigencias, a veces contradictorias, de una sociedad pluralista, abierta a valores concurrentes que asume posiciones muy diferenciadas frente a los objetivos y funciones del derecho.(Petev, 1995, p. 24)

La ecopedagogía se relaciona con la creación de "ciencia con conciencia" y el trabajo articulado entre las diferentes disciplinas, como lo propone Morin: "Sé que la humanidad necesita una política. Que esta política necesita una antroposociología. Que la 
antroposociología necesita articularse en la ciencia de la naturaleza, que esta articulación requiere una reorganización en cadena de la estructura del saber"(Morin, 1984, p. 37).

La ecopedagogía parte del principio transformador del ser humano, para que se pueda comunicar con otras culturas y con el medio ambiente ya que, solo quien es capaz de cuestionarse a sí mismo, podrá cuestionar las bases económicas y sociales identificadas por Warat como turbocapitalismo, consumismo y choque de culturas, y así interactuar con su entorno a partir de un nuevo paradigma ecológico.

\section{Ecociudadanía}

El objetivo de la ecología es crear mejores condiciones de existencia. La "ocupación ecológica" pasa por la tentativa de mejorar las condiciones ambientales, sociales y afectivas de la existencia. Un objetivo que solo se podrá cumplir si el hombre consigue individual y colectivamente, abrirse para una realidad de sentidos en permanente estado de multiplicidad. La ecociudadanía vista como un problema de vínculos que establecen los hombres: entre sí, con la naturaleza, de una sociedad para otra. (Morin, 1984, p. 37)

Es necesario educar ciudadanos con una nueva racionalidad ambiental: "La racionalidad ambiental es una utopía forjadora de nuevos sentidos existenciales; trae consigo una re significación de la historia, a partir de los límites y de las potencialidades de la condición humana, de la naturaleza y de la cultura" (Leff, 2004, p. 405).

\section{Transición hacia una sociedad ecológica}

Las acciones individuales tales como el reciclaje casero, el uso de productos ecológicos y medios de transporte tales como bicicleta, transporte público y autos híbridos son necesarias, aunque insuficientes frente a la magnitud de los desastres naturales y sociales que se presentan en la actualidad.

Vivimos en medio de una sociedad de riesgo global, que necesita tener estrategias locales para la conservación del medio ambiente global. [...] En la perspectiva realista, las consecuencias y peligros de la producción industrial desarrollada ahora "son" globales. Este "son" se apoya en hallazgos científicos y en los debates de la destrucción en curso (de la capa de ozono por ejemplo); el desarrollo de las fuerzas productivas está entretejido con las fuerzas destructivas $y$, en conjunto, ambas generan la 
novedosa dinámica del conflicto de una sociedad en riesgo global. (Beck, 2002, p. 35-36)

En consecuencia con la sociedad del riesgo global descrita por Beck, es necesario combinar las acciones individuales con las colectivas y las locales con acciones de nivel nacional, internacional y transnacional.

Como propuesta colectiva Escobar propone para Colombia:

[...] pensar en una verdadera transición ecológica y cultural hacia una sociedad muy diferente. Muchos visionarios nos hablan de las características de estas transiciones: la reestructuración de la producción de los alimentos con base en la descentralización, el cultivo orgánico y la biodiversidad; la democracia participativa; las autonomías locales; el uso menos intenso de los recursos; la reducción del consumo de energía y fuentes alternativas de ésta; y las economías sociales y solidarias. Pospetróleo, poscarbono, poscapitalismo, posextractivismo, posdesarrollo son algunos de los imaginarios emergentes. En sus formas más avanzadas, estas narrativas nos hablan de un cambio de modelo civilizatorio. (El Espectador, 2011, p. 14)

\section{CONCLUSIONES}

A pesar de los conflictos económicos, sociales y ambientales, que ubican nuestra realidad en medio de una "sociedad en riesgo", hemos elegido el 'futurible' de la emancipación ecológica como un escenario que nos permita imaginar un país, Colombia, comprometido con la salud, la vida y la supervivencia de la especie humana y de otras especies.

Para hacer realidad la emancipación ecológica hemos definido los pilares de la ecopedagogía, ecociudadanía y la transición hacia una sociedad ambiental, como tres conceptos que nos permitirán realizar una propuesta teórica para la protección del paisaje natural y cultural en el Triángulo del Café.

Retomando los lineamientos dados por Leff, Morin, Petev, y Warat, consideramos que la interdisciplinariedad se torna imprescindible para la educación de ciudadanos capaces de crear "ciencia con conciencia", "ecociudadanía" y "sociedades ecológicas".

En términos de ecociudadanía la democracia es un deber ser cultural multiexpresivo, y no solo un conjunto de garantías jurídicas. En palabras de Touraine: "Un espacio para reconciliar los distintos 
proyectos de vida". "Esto para mí significa: todo lo que preserva el encuentro con el otro, la cultura de la alteridad. La aceptación del otro como diferencia que permite la realización de las identidades" (Warat, 2004, p. 417).

A través de la educación, es entonces que podremos hacer realidad el 'futurible' de la emancipación ecológica, pero para ello la universidad deberá, a través de la práctica, interactuar con las comunidades indígenas y campesinas, entre otras, para así fortalecer la gobernanza ambiental.

Este diálogo a partir de la información y la igualdad entre las partes intervinientes: sociedad civil, academia, Estado, encuentra libertad de expresión a partir del fortalecimiento del poder local y de la conformación de redes para la investigación ambiental, en comunicación directa con otras redes internacionales.

Para lograrlo, seguimos creyendo que la construcción de políticas públicas y el Derecho, son los sistemas colectivos creados por el hombre, con la mayor posibilidad de hacer real la emancipación ecológica. Sin embargo, el verdadero reto para la política y el derecho, se encuentra en la innovación metodológica.

Es allí en donde se encuentran las mayores dificultades para la puesta en práctica que implica la interdisciplinariedad, el diálogo de saberes y comprender la posición del otro con el fin de llegar a consensos, teniendo como objetivo la conservación de las áreas protegidas, los resguardos indígenas y el patrimonio mundial existente en el Triángulo del Café.

\section{AGRADECIMIENTOS}

La autora agradece a la Universidad de Caldas por la financiación del trabajo de campo, a las comunidades indígenas y campesinas del Triángulo del Café, a los integrantes del Semillero de Derecho Ambiental (2007-2010); así como a la beca otorgada por el Ministerio de Educación de Brasil (Beca PEC-PG 2011-2015).

\section{REFERENCIAS}

- Beck, Ulrich. (2002). La sociedad del riesgo global. Madrid: Siglo XXI de España Editores. (Título original: World risk society).

- Correa, Diana Magally. (2009). Memorias de la VII Convención de Medio Ambiente. Disponible en: http://www.cubambiente.com/memorias/2009/02\%20VII\%20C ONGRESO\%20EDUCACION\%20AMBIENTAL- 
RESUMENES\%20Y\%20TRABAJOS.pdf (consulta realizada el 25 de julio de 2011).

- De Sousa, Boaventura y Rodríguez, César A. (Eds.).(2007).El derecho y la globalización desde abajo. Hacia una legalidad cosmopolita. México: Antrophos.

- El Espectador. (noviembre 29 de 2011). Colombia, entre los más afectados por el clima en el mundo. Redacción Ipad. Disponible

http://www.elespectador.com/noticias/nacional/articulo-

313969-colombia-entre-los-mas-afectados-el-clima-elmundo

- El Espectador. (Julio 20 de 2011).10 ideas para reconstruir a Colombia. ¿Qué se debe hacer para evitar una nueva tragedia ambiental? Coordinadores Pablo Correa y Carolina Gutiérrez. Entrevista con Margarita Astrálaga y Arturo Escobar. Disponible en: http://www.elespectador.com/noticias/nacional/articulo285708-10-ideas-reconstruir-colombia

- Guber, Rosana. (2001).La etnografía. Método, campo y reflexividad. Bogotá: Grupo Editorial Norma.

- Leff, Enrique. (2004).Saber Ambiental. Sustentabilidad, racionalidad, complejidad y poder (4a ed.). México, D.F.:Siglo XXI Editores, UNAM y PNUMA.

- Maya, Augusto Ángel. (1996).El Reto de la vida. Bogotá: Ecofondo.

- Morin, Edgar. (1984).Ciencia con conciencia. Barcelona: Anthropos.

- Organización de las Naciones Unidas. (1992).Convenio de Diversidad Biológica. Río de Janeiro.

- Organización Internacional del Trabajo. Convenio 169: sobre Pueblos Indígenas y Tribales en Países Independientes. Art. $5,6,7$.

- Petev, Valentin. (1995).¿Cuál es el método? La metodología jurídica al umbral del siglo XXI. Bogotá: Universidad Externado de Colombia.

- Red Alma Máter. (2010). Paisaje cultural cafetero. Obtenido el 25 de julio de 2011, desde http://www.almamater.edu.co/sitio/contenido-paisajecultural-cafetero-59.html

- _ (s.f.). Ecorregión Eje Cafetero. Obtenido el 15 de septiembre de 2008, desde http://www.almamater.edu.co/ecorregion_documentos.htm

- Warat, Luis Alberto. (2004).Territórios desconhecidos. A procura surrealista pelos lugares do abandono do sentido e da reconstrução da subjetividade. Por quem cantam as Sereias. Informe sobre Ecociudadanía. Gênero e Direito. Florianópolis: Fundación Boiteux.

1.Abogada con Maestría en Antropología. Estudiante del Doctorado en Ciencias Jurídicas UNIVALI, becaria CAPESPEC-PG, Brasil. Profesora de la Facultad de Ciencias 
Jurídicas y Sociales, Universidad de Caldas, Manizales, Colombia.diana.correa@ucaldas.edu.co

2.Para ampliar la información sobre la investigación sociojurídica, se sugiere consultar las actas realizadas por la Red de Investigaciones Sociojuridicas Nodo Eje Cafero, en el link: http://www.redsociojuridica.org/nodo_cafetero.htm, en especial, las actas 006 a 011, de septiembre de 2007 a octubre de 2008.

3.El método de Investigación Acción Participativa (IAP) fue desarrollado por el sociólogo colombiano Orlando Fals Borda. Para ampliar información sobre este método se recomienda leer: Fals Borda, Orlando. (1990). La Investigación: Obra de los Trabajadores. Aportes, 20, 9-16.

4.La etnografía es el método por excelencia de la antropología. Para la narración de los resultados de este artículo en torno a la reflexividad y la subjetividad, se empleó el texto: ¿Qué es la antropología? De Rosana Guber (2001).

5.La disminución de la producción de café también se encuentra acompañada de factores políticos, económicos y sociales, que han sido denunciados por las marchas y manifestaciones campesinas durante los años 2012 y 2013.

6.SPNN: Sistema de Parques Nacionales Naturales.

7."Artículo 63: Los bienes de uso público, los parques naturales, las tierras comunales de grupos étnicos, las tierras de resguardo, el patrimonio arqueológico de la Nación y los demás bienes que determine la ley, son inalienables, imprescriptibles e inembargables" (Constitución Política de Colombia, 1991).

8.Artículo 246 de la Constitución Política de Colombia.

9.SIRAP-EC: Sistema Regional de Áreas Protegidas Eje Cafetero.

10. CAR: Corporación Autónoma Regional. Autoridad ambiental descentralizada que pertenece al Ministerio del Ambiente.

11. WWF: World Wild Foundation. Fundación Mundial para la Conservación.

12. Título original en portugués "Porque cantam as sereias"; la traducción es nuestra.

Para citar este artículo: Correa, D. M. (2013). Ecopedagogía para preservar las áreas protegidas y el paisaje cultural cafetero. Revista Luna Azul, 37, 49-62. Recuperado de http://lunazul.ucaldas.edu.co/index.php?option=content\&task=view\&id=844 